\title{
Experimental study of 3.6-meter segmented-aperture telescope for geostationary Earth observation satellite
}

Yasutaka Fujii, Takeru Uno, Shigeru Ariki, Koya Suehiro, Shigetaka Itakura, et al.

Yasutaka Fujii, Takeru Uno, Shigeru Ariki, Koya Suehiro, Shigetaka Itakura, Masao Imaki, Tadahito Mizutani, Seichi Sato, Keiichi Yanagase, Susumu Yasuda, Taro Kawano, Toshiyoshi Kimura, "Experimental study of 3.6-meter segmented-aperture telescope for geostationary Earth observation satellite," Proc. SPIE 11852, International Conference on Space Optics - ICSO 2020, 118522G (11 June 2021); doi: 10.1117/12.2599379

SPIE Event: International Conference on Space Optics - ICSO 2021, 2021, Online Only 


\section{International Conference on Space Optics-ICSO 2020}

Virtual Conference

30 March-2 April 2021

Edited by Bruno Cugny, Zoran Sodnik, and Nikos Karafolas
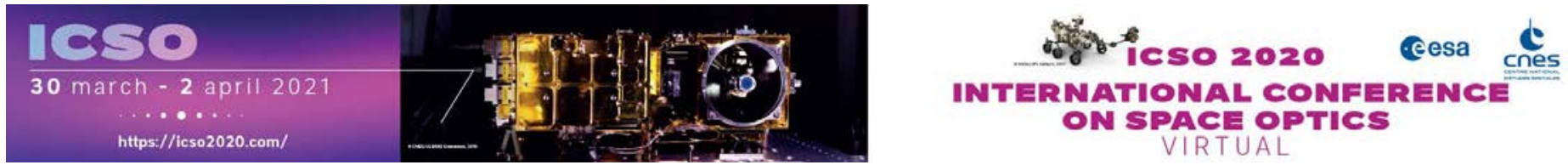

\section{Experimental study of 3.6-meter segmented-aperture telescope for geostationary Earth observation satellite}

\section{Cesa isoporesedings ecres}




\title{
Experimental study of 3.6-meter segmented-aperture telescope for geostationary Earth observation satellite
}

\author{
Yasutaka Fujii ${ }^{\mathrm{a}}$, Takeru Uno*a, Shigeru Ariki ${ }^{\mathrm{a}}, \mathrm{Koya}$ Suehiro $^{\mathrm{a}}$, Shigetaka Itakura ${ }^{\mathrm{a}}$, Masao Imaki ${ }^{\mathrm{a}}$, \\ Tadahito Mizutani $^{\mathrm{b}}$, Seichi Sato ${ }^{\mathrm{b}}$, Keiichi Yanagase ${ }^{\mathrm{b}}$, Susumu Yasuda ${ }^{\mathrm{b}}$, Taro Kawano ${ }^{\mathrm{b}}$, \\ Toshiyoshi Kimura ${ }^{\mathrm{b}}$ \\ ${ }^{a}$ Mitsubishi Electric Corporation, 325 Kamimachiya, Kamakura, Kanagawa, 247-8520 Japan; \\ bJapan Aerospace Exploration Agency, 2-1-1 Sengen, Tsukuba, Ibaraki, 305-8505 Japan.
}

\begin{abstract}
The imager that observes the ground surface with high spatial resolution from geostationary orbit has been studied. To obtain image data with high ground sampling distance (GSD) from a geostationary orbit, an extremely large-diameter and long-focal-length telescope is required. In order to realize this imager, we plan to adopt a synthetic aperture type reflection optical telescope (Korsch type) in which the primary mirror is composed of six segmented mirrors. The diameter of the synthesized aperture telescope is approximately $3.6 \mathrm{~m}$, and the focal length is approximately $30 \mathrm{~m}$. Prior to the development of the actual imager, we have been producing a full-scale prototype of the segmented mirror that constitutes the primary mirror and its supporting structure. The aperture shape of the prototype segmented mirror is hexagonal, and its diagonal length is approximately $1.35 \mathrm{~m}$. The supporting structure of the prototype segmented mirror incorporates a mechanism (6-axes adjustment mechanism) for adjusting the alignment of the segmented mirror with six degrees of freedom. A mechanism for adjusting the curvature of the segmented mirror (curvature adjustment mechanism) was also incorporated. In this paper, the design, the manufacturing, and the testing status of this prototype are described.
\end{abstract}

Keywords: Space Optics, Large Optics, Segmented-Aperture telescope, Lightweight mirror, Geostationary Satellite

\section{INTRODUCTION}

While many Earth observation imagers, including those for commercial use, provide satellite images with high spatial resolution less than one meter, the motivation for our research also focuses on improving the temporal resolution of imager systems. In the ideal observation, the satellite images can be obtained anywhere and anytime the users want without time delays. Although there would be technical limitations in reality, drastic improvements in the temporal resolution and the latency, which is defined as the data delivery time from the request for an observation, may lead to unprecedented satellite applications. Such a satellite imaging system would enhance resilience to natural disasters, which have become increasingly serious on a global scale in recent years, by reducing human suffering and damage to infrastructure.

There are two major strategies to improve the temporal resolution. One is to observe with a large number of satellite constellations, and many commercial services are under consideration. The other is to build an observation system from geostationary orbit. Since satellites in geostationary orbit can always observe the same ground area, the temporal resolution is ideally zero (continuous imaging), and since they can always communicate with a specific ground station, the latency can be minimized. On the other hand, the observation area is limited with a single satellite, and the distance of $36,000 \mathrm{~km}$ is a disadvantage in terms of spatial resolution. The tentative specifications of the geostationary Earth observation satellite are summarized in the Table 1.

Authors have been investigating an imaging satellite that can observe around Japan with a resolution of less than 10 meters in panchromatic mode ${ }^{1}$. In order to achieve such a resolution, a large aperture telescope is required, and the feasibility of a segmented telescope system was investigated ${ }^{2}$. Since the geostationary high-resolution imager will be operated in geostationary orbit, the distance to the Earth's surface will be very large, and a telescope with an extremely large aperture and long focal length will be required for high-resolution observations. For the geostationary highresolution imager, a synthetic aperture type reflective optical system (Korsch type) with a primary mirror composed of multiple segmented mirrors is envisioned to realize this telescope. 
Table 1. The specifications of the geostationary Earth observation satellite (Current Assumption).

\begin{tabular}{|c|c|}
\hline Item & Specification \\
\hline Imaging Method & Area sensor imaging \\
\hline Imaging Mode & Images / Videos \\
\hline Spectral Band & $\begin{array}{l}\text { Panchromatic : } 1 \text { band } \\
\text { Multi-Spectral : } 6 \text { band } \\
\text { Infrared (IR) : } 1 \text { band }\end{array}$ \\
\hline $\begin{array}{l}\text { Ground Sampling } \\
\text { Distance (GSD) }\end{array}$ & $\begin{array}{l}\text { Panchromatic: } 5-10 m \\
\text { Multi: } 15-30 m \\
\text { IR::TBD }\end{array}$ \\
\hline Field of View & $100 \times 100 \mathrm{~km}$ (All band) \\
\hline
\end{tabular}

There is no precedent of adopting a segmented telescope for Earth observation satellites to the extent of our knowledge except for James Webb Space Telescope which is an astronomical telescope about to be launched. Therefore, a prototype evaluation of the key technology was planned prior to full-scale satellite development in order to identify and mitigate potential technological risks. Figure 1 shows an image of the segmented telescope for the geostationary high-resolution imager. The telescope is a Korsch three-mirror system with a synthetic focal length of about 30 meters, and a field of view of 0.2 degrees. The primary mirror consists of six hexagonal segmented mirrors. The diameter of the circle circumscribed by the synthesized aperture of the entire primary mirror is about 3.6 meters. The telescope has a diameter of about 4 meters and a height of about 7 meters including the structures. These tentative specifications are summarized in the Table 2. Prior to the development of the geostationary high-resolution imager, the segmented mirror that constitutes the primary mirror and a set of its support structures were prototyped and evaluated on a scale equivalent to that of the actual system (full scale). In this paper, the design, the manufacturing, and the testing status of this prototype is described.

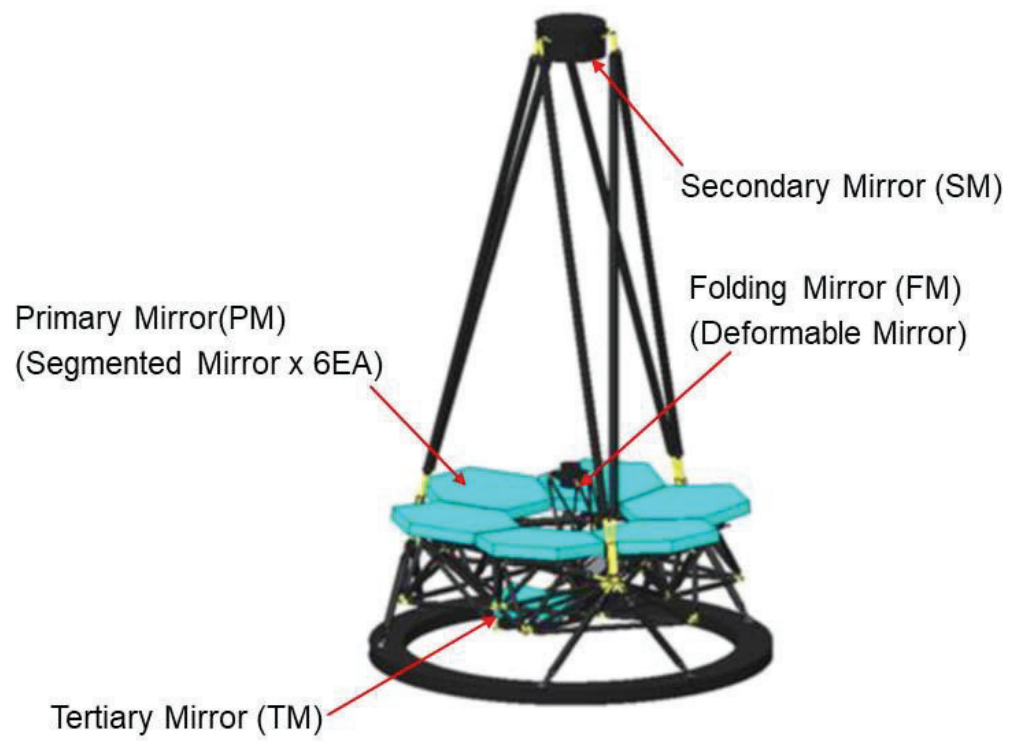

Figure 1. Image of the telescope for the geostationary high-resolution imager. 
Table 2. Telescope Optics Specifications (Current Assumption)

\begin{tabular}{l|l}
\multicolumn{1}{c|}{ Item } & \multicolumn{1}{c}{ Specification } \\
\hline Type & $\begin{array}{l}\text { Segmented-Aperture Telescope } \\
\text { (6 Hexagonal segments) }\end{array}$ \\
\hline Telescope design & Korsch type Reflected optics \\
\hline Aperture & $3.6 m$ approx. \\
\hline Focal Length & $30 \mathrm{~m}$ approx.(tentative) \\
\hline Field of View (FOV) & $0.2 \times 0.2 \mathrm{deg}$ \\
\hline Dimension & $\varphi 4 \times 7 \mathrm{~m}$ (tentative) \\
\hline $\begin{array}{l}\text { Adjustment } \\
\text { Mechanism } \\
\text { (candidate) }\end{array}$ & - - Curvature adjustment mechanism on PM \\
& - Adjustment mechanism on SM \\
\hline
\end{tabular}

\section{DESIGN OF THE FULL-SCALE MODEL}

Figure 2 (left) shows the configuration of a full-scale model of the segmented mirror ASSY and its support structure. Figure 2 (right) shows the structure of segment mirror Sub-ASSY with bipods, 6-axes adjustment mechanisms, and curvature adjustment mechanisms.
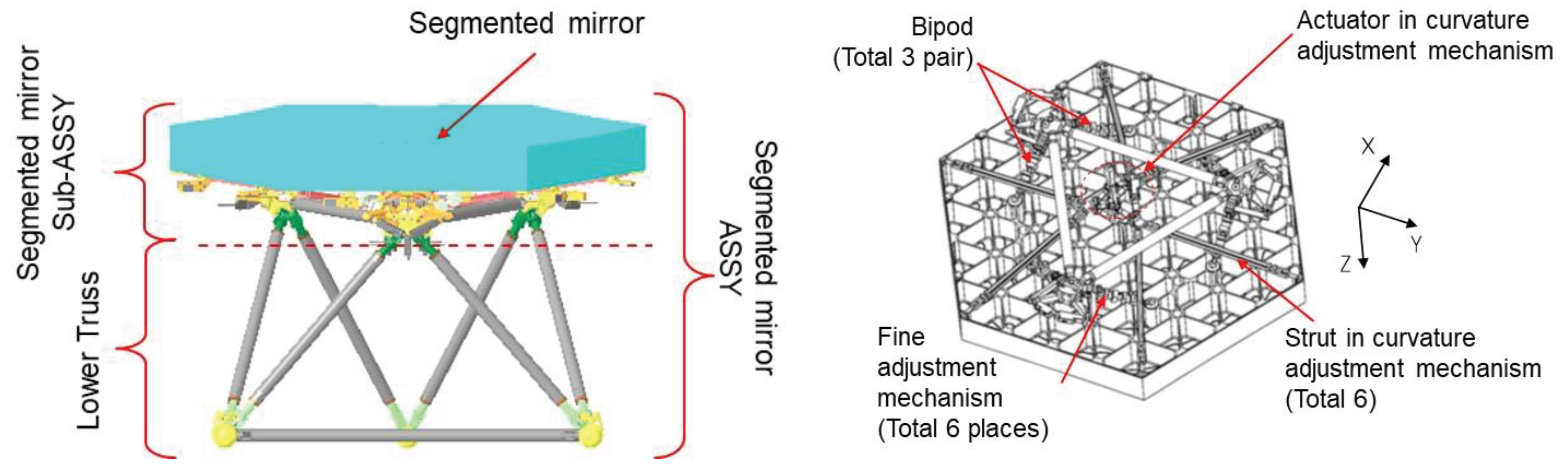

Figure 2. the configuration of the full-scale model. (left): The Segmented mirror ASSY; (right): The Segmented mirror SubASSY. The segmented mirror ASSY includes the segmented mirror Sub-ASSY and lower truss. The segmented mirror SubASSY consists of a segmented mirror, bipods, 6-axes adjustment mechanisms, and curvature adjustment mechanisms.

Based on previous studies ${ }^{3,4}$, cordierite, a ceramic material with zero expansion at room temperature and high specific rigidity, was selected as the substrate for the segmented mirror. The diameter of the circle circumscribed on the hexagonal aperture of the segmented mirror is about $1.3 \mathrm{~m}$, and the radius of curvature is about $11 \mathrm{~m}$. This is a large aperture and long radius of curvature for a single mirror. The backside of the segmented mirror was pocketed to reduce its weight significantly. The shape of the segmented mirror is illustrated in Figure 3. The specifications of the segmented mirror are shown in Table 3. 

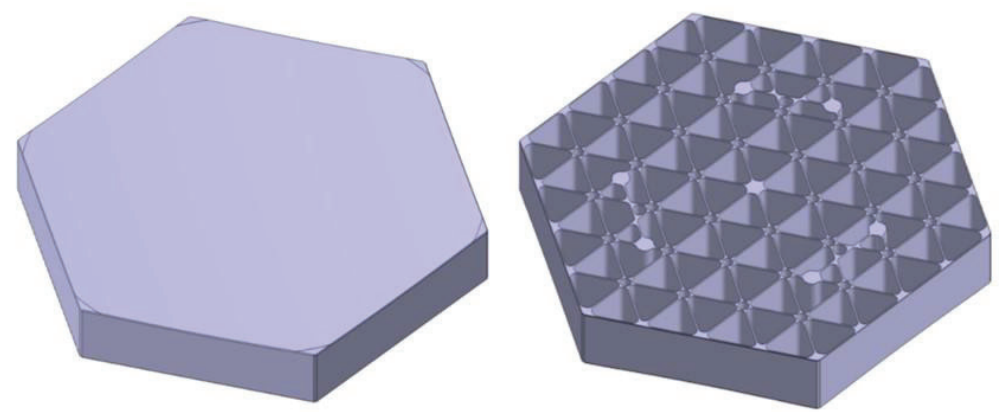

Figure 3. The shape of segmented mirror (left: the surface side; right: the back side). Significant light weighting was achieved due to pocket machining on the back side of the mirror

Table 3. The specifications of the segmented mirror. In this prototype, the shape of mirror surface was spherically designed for the ease of inspection and considering the manufacturing period, the surface accuracy was designed within the extent that the surface deformation can be verified with high accuracy.

\begin{tabular}{|c|c|c|}
\hline \multirow[b]{2}{*}{ Item } & \multicolumn{2}{|c|}{ Specification } \\
\hline & prototype & $\begin{array}{l}\text { Flight model } \\
\text { assumption }\end{array}$ \\
\hline Material & \multicolumn{2}{|l|}{ Cordierite ceramics } \\
\hline Aperture Shape & \multicolumn{2}{|l|}{ Hexagon } \\
\hline Aperture diameter & \multicolumn{2}{|c|}{$\begin{array}{l}\text { Outer }: 1.35 \mathrm{~m} \text { approx. } \\
\text { Clear Aperture }: 1.3 \mathrm{~m} \text { approx. }\end{array}$} \\
\hline $\begin{array}{l}\text { Shape of mirror } \\
\text { surface }\end{array}$ & Spherical surface & Aspherical surface \\
\hline Curvature radius & \multicolumn{2}{|l|}{$11 m$ approx. } \\
\hline Surface accuracy & $63 \mathrm{~nm} \mathrm{rms}$ or lower & Better than prototype \\
\hline Surface roughness & $2 \mathrm{~nm}$ rms or lower & \\
\hline
\end{tabular}

The segmented mirror was supported on the holding structure via three sets of bipods. In order to reduce the distortion generated by the lower truss (see Figure 2), each pod has a plate spring to mitigate the bending moment. On the assumption that the apertures of the six segmented mirrors will be synthesized in the flight system, the following adjustment mechanisms, which will be intended to be used for this adjustment, were incorporated in the support structures of the segmented mirror:

The six-axes adjustment mechanisms (coarse adjustment mechanisms / fine adjustment mechanisms).

The curvature adjustment mechanisms.

The six-axes adjustment mechanisms are the mechanisms to adjust the position of the segmented mirror in three degrees of freedom for translation and three degrees of freedom for rotation. It consists of two types of mechanisms, the coarse adjustment mechanisms, and the fine adjustment mechanisms, to achieve both a wide adjustment range and high adjustment resolution.

The coarse adjustment mechanisms are used to adjust the position of the segmented mirror over a wide range. It is intended to be used for assembly and adjustment of the telescope on the ground. Adjustment is made by changing the length of the six rods on the lower truss (the rods that connect the segmented mirror side to the opposite side) (Figure 4). The length of the rods is changed by rotating a turnbuckle-like manual mechanism built into each rod. 

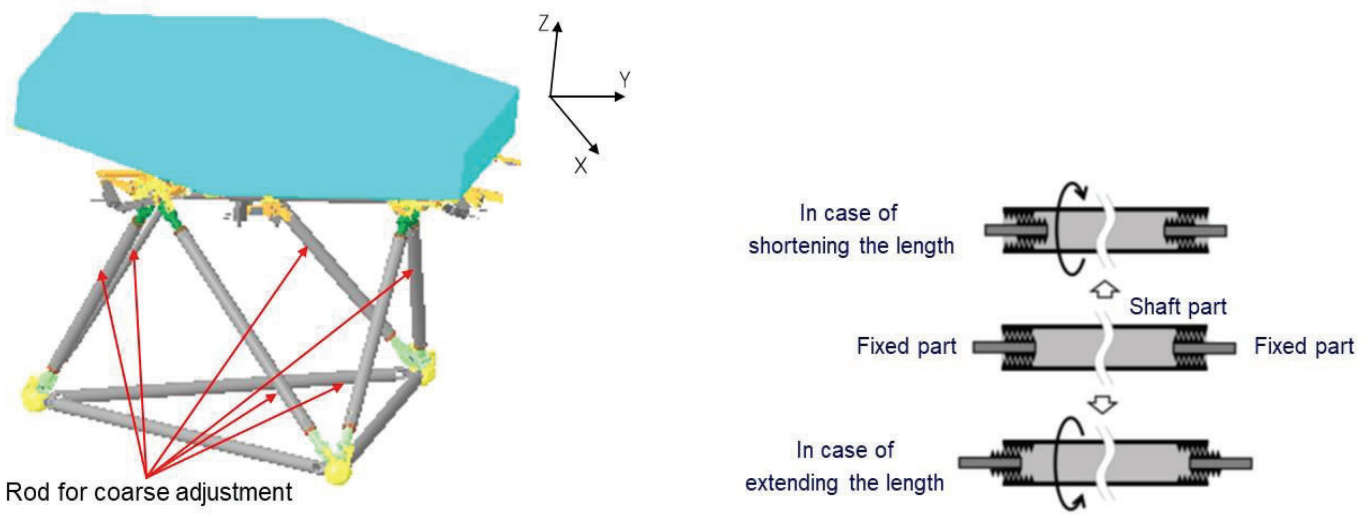

Figure 4. The coarse adjustment mechanisms in the six rods on the lower truss (left). A schematic diagram of rod length adjustment (right).

The fine adjustment mechanisms are the mechanisms to adjust the position of the segmented mirror with high resolution. It is intended to be used for detailed performance adjustment of the telescope on the ground and in orbit. The adjustment is performed by displacing the end of the 6 pods $(2 \times 3$ pairs $)$ supporting the segmented mirror in the axial direction using a linear actuator. The linear actuator was equipped with a displacement reduction mechanism, which enables fine displacement adjustment.

The curvature adjustment mechanism is used to adjust the curvature of the segmented mirror. In the flight system, it will be used on the ground and in orbit to correct the variation of curvature between the mirrors and to keep the surface shape error of the synthesized entire primary mirror within a desired range. The adjustment is performed by pushing and pulling each corner of the segmented mirror through six struts (see Figure 2). The force required for pushing and pulling is adjusted by actuators that incorporate a mechanism similar to the fine adjustment mechanisms.

The major specifications of the six-axes adjustment mechanisms and the curvature adjustment mechanisms are summarized in Table 4.

Table 4 The major specifications of the six-axes adjustment mechanisms and the curvature adjustment mechanisms.

\begin{tabular}{|c|c|c|}
\hline \multicolumn{2}{|c|}{ Item } & Specification \\
\hline \multicolumn{2}{|c|}{ Mirror support structure } & Inverted bipod \\
\hline \multicolumn{2}{|c|}{ Adjustment mechanism } & $\begin{array}{l}\text {-6-axes adjustment mechanism (fine adjustment) } \\
\text { - Curvature adjustment mechanism }\end{array}$ \\
\hline \multirow[t]{2}{*}{$\begin{array}{l}\text { Coarse adjustment } \\
\text { mechanisms }\end{array}$} & Resolution & $\begin{array}{l}\triangle X Y Z: 10 \mu m \text { or lower } \\
\theta X Y Z: 10 " \text { or lower }\end{array}$ \\
\hline & Range & $\begin{array}{l}\Delta X Y Z: \pm 10 \mathrm{~mm} \text { or upper } \\
\theta X Y: \pm 25^{\prime} \text { or upper, } \theta Z: \pm 50^{\prime} \text { or upper }\end{array}$ \\
\hline \multirow[t]{2}{*}{$\begin{array}{l}\text { Fine adjustment } \\
\text { mechanisms }\end{array}$} & Resolution & $\begin{array}{l}\Delta X Y: 0.1 \mu \mathrm{m} \text { or lower, } \Delta Z: 20 \mathrm{~nm} \text { or lower } \\
\theta X Y: 0.1 " \text { "or lower, } \theta Z: 0.5^{\prime \prime} \text { or lower }\end{array}$ \\
\hline & Range & $\begin{array}{l}\Delta X Y: \pm 25 \mu \mathrm{m} \text { or upper, } \Delta \mathrm{Z}: \pm \text { TBD or upper } \\
\theta \mathrm{XY}: \pm 25 \text { "or upper, } \theta Z: \pm 50 \text { "or upper }\end{array}$ \\
\hline \multirow{2}{*}{$\begin{array}{l}\text { Curvature } \\
\text { adjustment } \\
\text { mechanisms }\end{array}$} & Resolution & $20 \mathrm{~nm}$ rms or lower \\
\hline & Range & \pm TBD or upper \\
\hline
\end{tabular}




\section{STATUS OF MANUFACTURERING AND EVALUATION}

The outline of the manufacturing and evaluation plan for this prototype is shown in Figure 5.

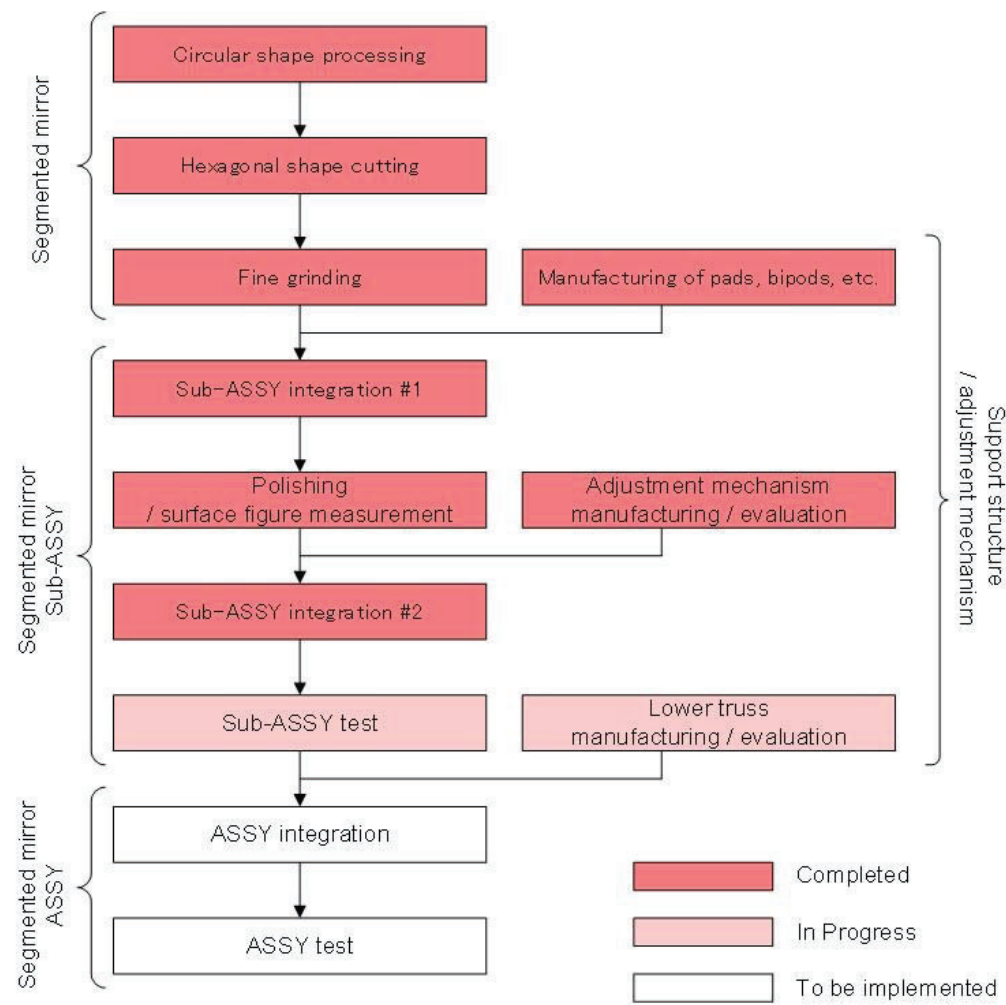

Figure 5. The outline of the manufacturing and evaluation plan for this prototype.

As the stage of "circular shape processing" in Figure 5, the appearance of the segmented mirror before sintering (after lightweight processing) is shown in Figure 6 (left). Figure 6 (right) shows the appearance of the segmented mirror at the stage when the "circular shape processing" was completed. The pockets on the backside of the segmented mirror were machined before the sintering of the ceramics. By machining in the soft state before sintering, machining efficiency was improved, and processing was completed in a short period. After sintering, hexagonal cutting process was performed (Figure 7).
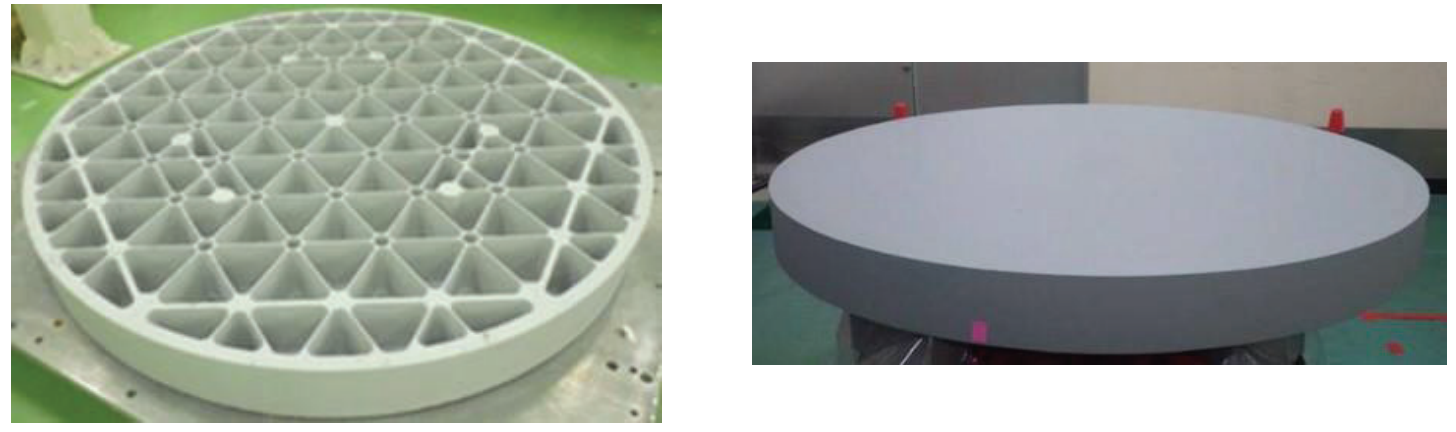

Figure 6. Appearance of the segmented mirror at the stage of "circular shape processing". (left): stage prior to ceramic sintering (after lightweight processing). (right): stage when the "circular shape processing" was completed. 


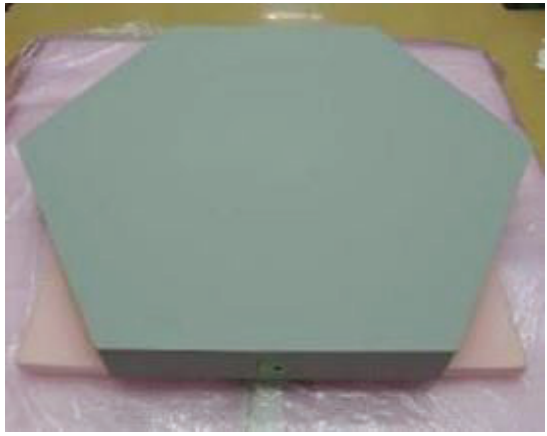

Figure 7. Appearance of the segmented mirror after hexagon shape cutting and fine grinding.

The process up to fine grinding was carried out in the form of a segmented mirror without bipods and lower truss. The Sub-ASSY was assembled by installing the bipods and other support structures to the segmented mirror after fine grinding was completed (Sub-ASSY integration \#1 in Figure 5). Figure 8 shows the completed Sub-ASSY (the protective cover was attached to the mirror surface). In parallel with the work on the segmented mirror, preliminary evaluations of each adjustment mechanism to be incorporated into the support structure were conducted. The results were reflected in the design of each adjustment mechanism incorporated in the segmented mirror assembly.
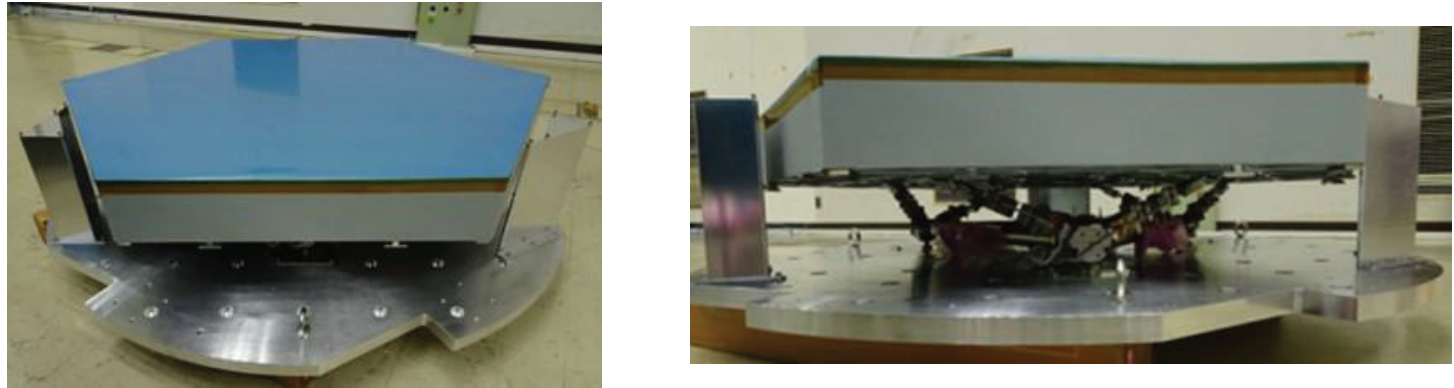

Figure 8. The completed Sub-ASSY with the protective cover. (left): top view. (right): side view.

The mirror was polished in the step of the segmented mirror Sub-ASSY (Figure 9; left). After completion of the polishing, the six-axes adjustment mechanisms and the curvature adjustment mechanisms were assembled into the segmented mirror Sub-ASSY as shown in Figure 9 (right) to complete the segment mirror ASSY.
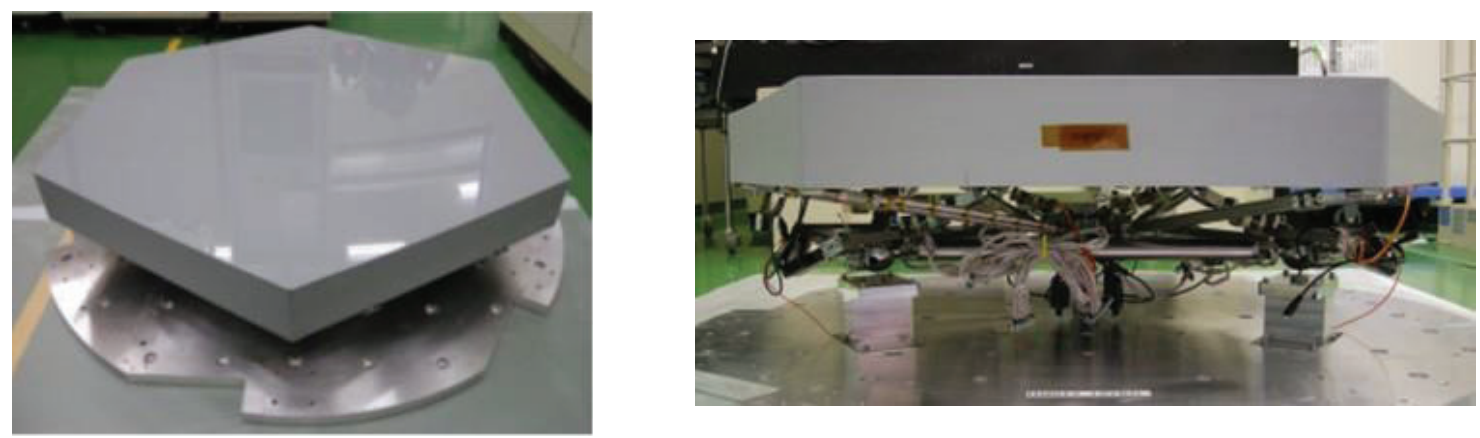

Figure 9. The segmented mirror Sub-ASSY after polishing. (left): Appearance of after polishing (the configuration of the surface figure measurement). (right): side view with the 6-axes adjustment mechanisms and the curvature adjustment mechanisms after Sub-ASSY integration \#2. 
A series of Sub-ASSY tests including environmental tests are in progress. The following items of the Sub-ASSY tests have been completed: gravity deformation evaluation test; thermal deformation evaluation test; 6-axes adjustment mechanism operation check test (shown in Figure 10, left); curvature adjustment mechanism operation check test; and thermal cycle test (shown in Figure 10, rihgt). The rest item of the Sub-ASSY tests is vibration test (at the time of writing this paper).

The segmented mirror ASSY integration and the ASSY test (gravity deformation test with lower truss) is planned to be performed.
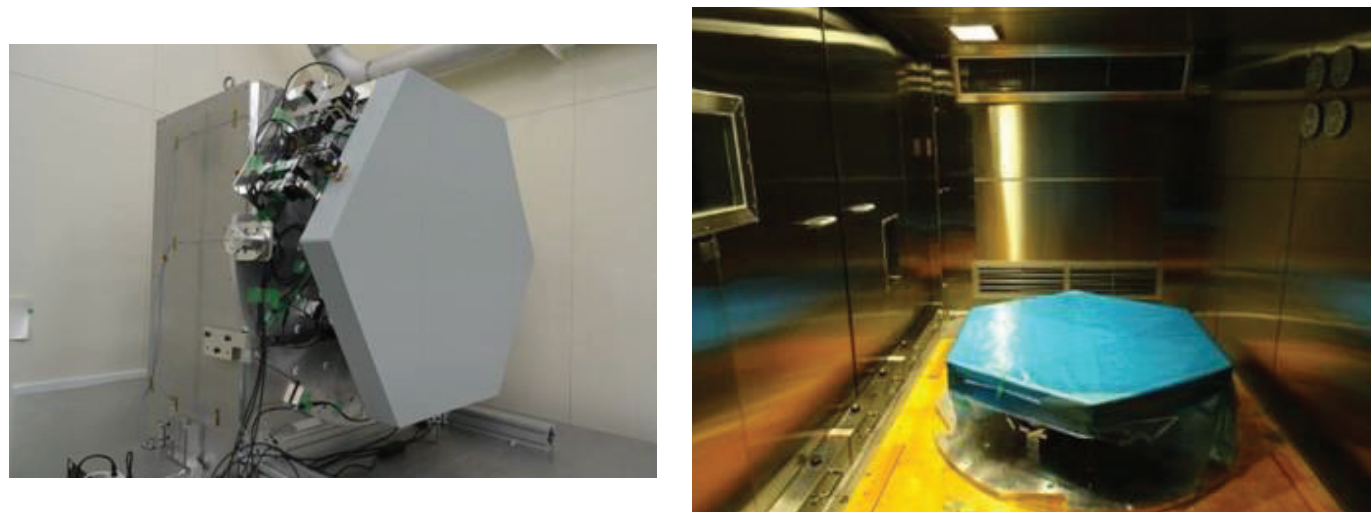

Figure 10. The Sub-ASSY test configuration. (left): 6-axes adjustment mechanism operation test. (right): Thermal cycle test.

During the polishing, surface shape was measured by using interferometer. Measurement attitude was $\mathrm{X}+1 \mathrm{G}$. Table 5 shows the results of surface shape measurement during polishing (without power component). The gravity deformation was analyzed (B) and removed from the results of $\mathrm{X}+1 \mathrm{G}$ measurement (A). Surface error at final polishing was $51 \mathrm{~nm}$ rms ( $0 \mathrm{G}$ conversion; $\mathrm{C})$, which was compliant to specification of surface accuracy in Table 3.

Table 5. Segmented mirror Sub-ASSY surface shape measurement results (during polishing).

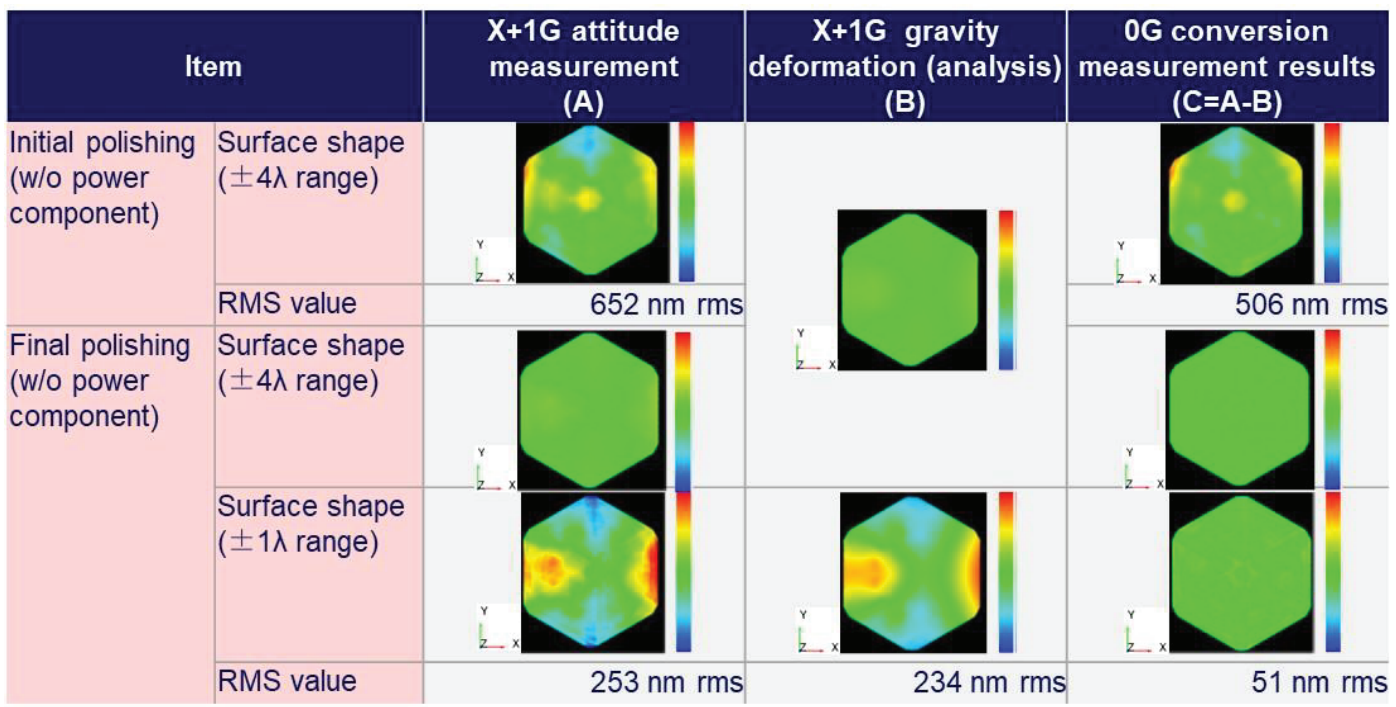


In order to realize the segmented-aperture telescope, it is essential to adjust the curvature of each segmented mirror. Absolute shape measurement with power (curvature) component was performed. The attitude of measurement was Z-1G. Absolute surface shape measurement (include power component) is shown in Table 6. The same measurement was performed before and after the polishing to evaluate the change of the mirror curvature due to polishing. In this prototype, the curvature was not actively changed by polishing. The trend of curvature error was consistent before and after polishing and the change in radius of curvature was $-0.006 \%$. This result confirms that the unintended change in curvature due to polishing was small.

Table 6. Segmented mirror Sub-ASSY absolute surface shape measurement results (before and after polishing).

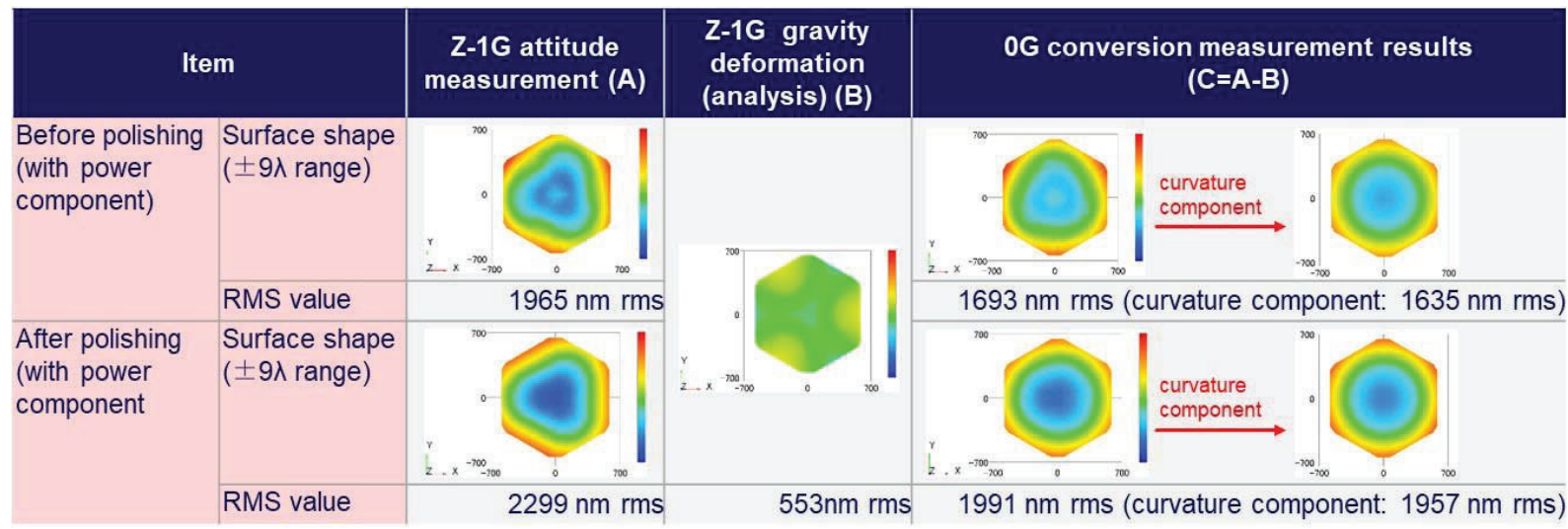

\section{CONCLUSION}

The full-scale production of the prototype and its evaluation was presented as to a set of segmented mirror and support structure (one segment) for geostationary high-resolution imager. The segmented mirror Sub-ASSY integration and the Sub-ASSY tests have been almost completed. The segmented mirror ASSY integration and ASSY test will be performed to get insight required for flight model of the geostationary high-resolution imager and to build foundation for future development.

\section{REFERENCES}

[1] Kimura et al.: Geostationary Earth observation satellite with large segmented telescope, Proc. IGARSS 2019, FR4.R4.5, 2019.

[2] Mizutani et al:: Conceptual study of 3.5-meter segmented mirror for geostationary Earth observation satellites, Proc. SPIE 10781, 1078117, 2018.

[3] Kamiya et al.: Development of ultra-lightweight and thermally-stable cordierite ceramic mirrors, Proc. SPIE, 10706, 107060O, 2018.

[4] T. Kamiya, S. Yasuda, A. Okamoto, S. Sato, H. Ueno, and T. Mizutani "Material selection of 3.5-meter segmented mirror for geostationary Earth observation satellite", Proc. SPIE 11116, 111160R (2019). 